\title{
Anisotropy of Brownian motion caused only by hydrodynamic interaction with a wall
}

\author{
Peter Holmqvist, Jan K. G. Dhont, and Peter R. Lang \\ Forschugszentrum Jülich, Institut für Festkörperforschung, Soft Matter Division, D-52425 Julich, Germany \\ (Received 31 March 2006; revised manuscript received 23 June 2006; published 3 August 2006)
}

\begin{abstract}
The diffusivity of spherical colloidal particles close to a planar hard wall is studied by dynamic light scattering with evanescent illumination. A novel setup allows us to independently vary the scattering vector components parallel $Q_{\|}$and normal $Q_{\perp}$ to the wall. An expression for the initial decay rate $\Gamma$ of the time autocorrelation functions is derived as a function of both $Q_{\|}$and $Q_{\perp}$, as well as the penetration depth of the evanescent wave, where hydrodynamic interactions of particles with the wall are included. This makes it possible to study the viscous wall drag effect quantitatively for particles as small as $85 \mathrm{~nm}$ in radius.
\end{abstract}

DOI: 10.1103/PhysRevE.74.021402

PACS number(s): 82.70.Dd, 68.35.Fx, 78.35.+c

In the past two decades dynamic light scattering with evanescent illumination (EWDLS) has been established to study Brownian motion of particles in the close vicinity of a planar solid interface. The diffusion of colloidal particles [1-5] has been studied as well as the translational and rotational dynamics of stiff polymers adsorbed to the interface [6] and the collective motion of end grafted polymer brushes [7,8]. However, all experimental studies on Brownian motion near walls by means of light scattering published so far suffer from the fact that (i) it has not been possible to measure the particle mobility parallel and normal to the wall independently and (ii) interpretation of the time autocorrelation function (TCF) is hampered by the lack of a theory for the form of the TCF that includes hydrodynamic interactions of the particles with the wall. We have set up an instrument for EWDLS with which we can change the scattering vector components parallel $Q_{\|}$and normal $Q_{\perp}$ to the wall separately. Consequently the diffusivities parallel and perpendicular to the interface are experimentally accessible. Further we derived an exact expression for the short-time behavior of the TCF including hydrodynamic interaction, which enables an unambiguous interpretation of the experimental correlation functions.

The experimental intensity time autocorrelation function (TCF) for evanescent wave scattering from hard spheres near a wall is significantly different from that of hard spheres in bulk. This difference was explained by the combination of a wall mirror effect and the particular illumination profile of the evanescent wave [1]. However, hydrodynamics predict that on top of this the diffusivity of spherical particles should decrease and become anisotropic if the colloids come closer to a planar wall than approximately the tenfold of their radius [9-11]. The diffusivities parallel and normal to the wall, at a distance $z$ from the wall, can be written as $D_{\|}(z)=D_{0} / \Lambda_{\|}(z)$ and $D_{\perp}(z)=D_{0} / \Lambda_{\perp}(z)$. Here $D_{0}$ is the bulk diffusion coefficient. The hydrodynamic mobilities $1 / \Lambda_{\|}$and $1 / \Lambda_{\perp}$ are functions of the ratio of the particle radius and the particle-to-wall separation distance $z$. The theoretically predicted behavior of the diffusivities is shown in Fig. 1. For macroscopic, i.e., non-Brownian particles, the prediction for $1 / \Lambda_{\perp}$ has been proven valid $[12,13]$ in sedimentation measurements of large spheres in highly viscous liquids. For particles that are small enough to perform Brownian motion, contradicting results can be found in literature. Using
Faxén's and Brenner's results [9-11], Garnier and Ostrowsky [2] calculated expressions for the diffusivities parallel and normal to a wall, but they could not distinguish them experimentally. Feitosa et al. [3] did not find evidence for a viscous wall drag effect in EWDLS experiments. Several other authors report anisotropic mobility of colloids close to walls, but it is not clear in any case whether this is due to viscous drag or to other effects influencing the particle mobility. Kihm et al. showed [14] in a three-dimensional (3D) particle tracing experiment that the mobility of a sphere with a radius of $100 \mathrm{~nm}$ is five to six times higher in the direction parallel to the wall as compared to the normal direction. However, this anisotropy is three times larger than predicted by Brenner, and the authors conjecture that it is very likely due to the electric double-layer repulsion between the particles and the wall. In a later contribution [15] the same authors find good agreement with theory for the particle mobility parallel to the interface, while the normal component is smaller than predicted. Lin et al. [16] reported a video microscopy study of particles with a diameter of $930 \mathrm{~nm}$, in which they show unambiguously that the particle diffusivity is slowed down and becomes anisotropic in the vicinity of a glass wall. Although the authors took some effort to density match the particles and exclude a gravity effect, this cannot be completely excluded, since these particles gain approximately $\Delta \phi_{\text {grav }} \approx 0.06 k_{B} T$ when sedimenting over a distance equal to their diameter. Hosoda and coworkers [4] found the same

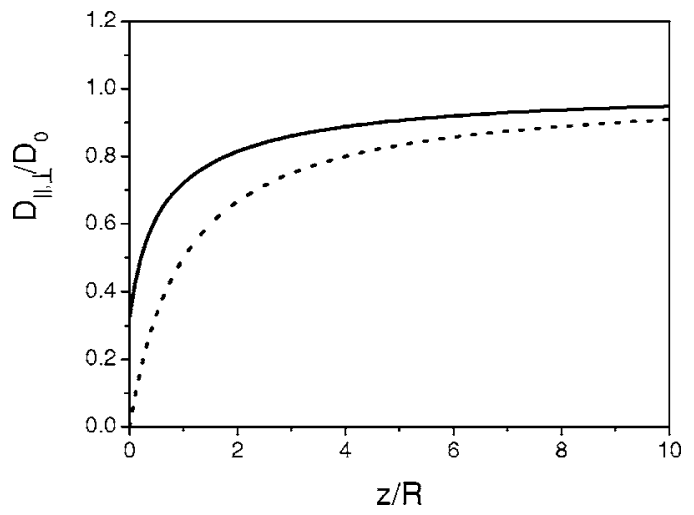

FIG. 1. Theoretical predictions for the distance dependence of the diffusivity of spherical particles parallel (full line) [9] and normal (dotted line) $[10,11]$ to a wall. 


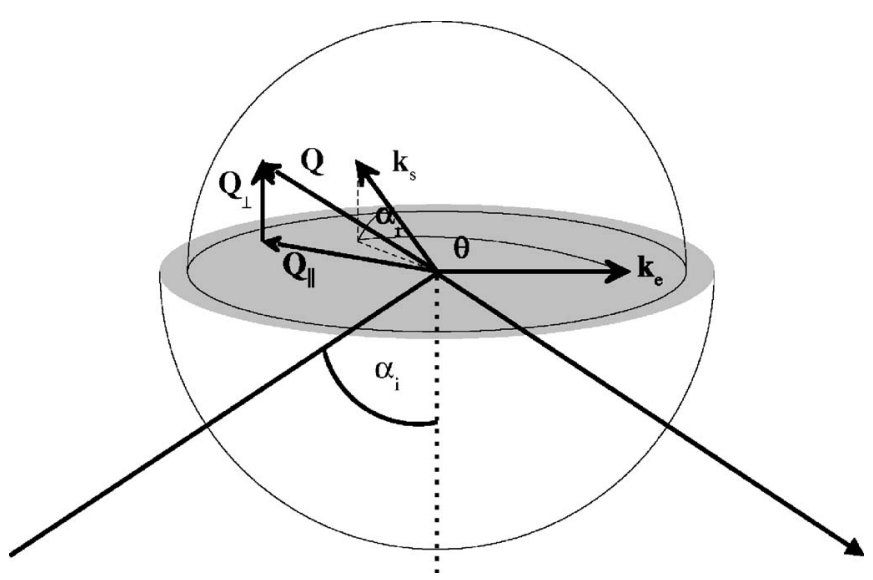

FIG. 2. Spherical sample cell with total scattering vector $\mathbf{Q}$ and scattering vector components parallel $\mathbf{Q}_{\|}$and normal $\mathbf{Q}_{\perp}$ to the reflecting interface.

result for a very similar system using EWDLS. However, for smaller particles their data do not allow to distinguish between bulk and near-wall dynamics. This controversy is mainly due to the fact that it was not possible to measure the diffusivity of Brownian hard spheres parallel and normal to a planar wall separately. In this contribution we show that the wall drag effect can be studied quantitatively for particles as small as $R=85 \mathrm{~nm}$ if, an EWDLS setup is employed, which allows to vary the magnitude of the scattering vector parallel $Q_{\|}$and normal $Q_{\perp}$ to the interface independently. Further, a theoretical expression for the time autocorrelation function is needed, which includes the effect of viscous drag.

In our laboratory we use a triple axis goniometer and a spherical sample cell to construct a scattering geometry as sketched in Fig. 2. The cell consists of a massive SF10-glass semispherical lens covered by a dome which contains the sample solution. The incident beam is totaly reflected from the interface between the bottom part and the solution, if the angle of incidence $\alpha_{i}$ is larger than the critical angle of total reflection $\alpha_{c}$. Since we can change the observation angle in-plane $\theta$ and off-plane $\alpha_{r}, Q_{\perp}=2 \pi \sin \alpha_{r} / \lambda$ can be changed while $Q_{\|}=2 \pi \sqrt{1+\cos \alpha_{r}^{2}-2 \cos \alpha_{r} \cos \theta} / \lambda$ is kept constant and vice versa. Here, $\lambda$ is the wavelength of the evanescent wave and the scattered light in the sample, and the total scattering vector magnitude is given by $Q=\sqrt{Q_{\perp}^{2}+Q_{\|}^{2}}$. In our experiments the illuminating beam comes from a He-Ne laser with a power of $35 \mathrm{~mW}$ and a vacuum wavelength of $\lambda_{0}=632.8 \mathrm{~nm}$, which is mounted on the source arm of the triple axis goniometer. Scattered light is collected with a single mode fiber and detected with an avalanche diode (ALV-APD, ALV-Laservertriebsgesellschaft, Germany) The diode's transistor-transistor logic (TTL) output is correlated with an ALV-6010 multiple tau correlator. Varying the angle of incidence, the penetration depth of the evanescent wave $2 / \kappa=\lambda_{0}\left(2 \pi \sqrt{\left(n_{1} \sin \alpha_{i}\right)^{2}-n_{2}^{2}}\right)^{-1}$ can be changed approximately in the range $100 \mathrm{~nm}<2 / \kappa<1 \mu \mathrm{m}$. The upper limit is mainly set by focusing of the incoming beam in the bottom part of the sample cell and the divergence of the penetration depth at the critical angle. The nominal critical angle of total reflection $\alpha_{c}$ can be calculated from the refractive index of the SF10 glass $n_{1}=1.7231$ and of the solution $n_{2}=1.3456$ for the sample solution as $\alpha_{c}=\arcsin \left(n_{2} / n_{1}\right)=50.52^{\circ}$.

Experiments were performed on charge-stabilized Polystyrene latex spheres with radius $R=85 \mathrm{~nm}$ in a $10 \mathrm{mM} / \mathrm{L}$ $\mathrm{NaCl}$ solution which was contained in the upper half-sphere of the sample cell. The Debye screening length is calculated from the salt concentration as $3 \mathrm{~nm}$. The particle volume fraction was adjusted to $2 \times 10^{-4}$. At this concentration no influence of the concentration on the bulk diffusion constant $D_{0}=2880 \mathrm{~nm}^{2} \mathrm{~ms}^{-1}$ was observed in conventional light scattering, while the scattered intensity was sufficiently high for EWDLS measurements. Under these conditions, the colloids may be regarded as hard spheres to a good approximation, because the average interparticle distance is of the order of $2000 \mathrm{~nm}$, which is large compared to the Debye length. Interaction with the wall, which can never be completely excluded, needs a more detailed discussion. At the given salt concentration the particles will be effectively expelled from the solid-solution interface up to a distance of approximately $10 \mathrm{~nm}$ by an extremely steep electrostatic potential. On the other hand, a conservative estimate shows that at $z=10 \mathrm{~nm}$, van der Waals attractions between the particles and the glass may amount to about $0.1 k_{B} T$. It cannot be excluded that these interactions will have an impact on the particle dynamics, but the effect is beyond the resolution of our experiment because the experiment probes mean particle mobilities averaged over the scattering volume. Although particles that are close to the wall will scatter more strongly than others further away, the contribution to the total scattering of the particles interacting with the wall can be neglected. First because the portion of the scattering volume where interaction with the wall is effective is about $10 \%$ at the smallest $(2 / \kappa=155 \mathrm{~nm})$ and less than $3 \%$ at the highest penetration depth $(2 / \kappa=551 \mathrm{~nm})$. Second, the particles will be repelled from the major part of this volume by electrostatic repulsion. Consequently our experiment will only probe the dynamics of particles that do not interact with the wall neither by electrostatic nor by dispersion forces. Experimental intensity correlation functions $g_{2}(t)$, obtained at the largest and the smallest penetration depth investigated, are shown in the inset of Fig. 3. These curves were recorded at fixed scattering vector components $Q_{\|}=0.01145 \mathrm{~nm}^{-1}$ and $Q_{\perp}=0.01316 \mathrm{~nm}^{-1}$. The field correlation functions, $g_{1}(t)$, the initial part of which is shown in the main plot of Fig. 3, were calculated using the standard relation

$$
g_{2}(t)=1+2 C_{1} g_{1}(t)+\left[C_{2} g_{1}(t)\right]^{2}
$$

for the case of mixed homodyne and heterodyne detection. Here $C_{2}=1-\sqrt{1-A}, C_{1}=C_{2}-C_{2}^{2}$, and $A$ is the intercept of $g_{2}(t)$. These curves illustrate that the initial relaxation rate $\Gamma$ of $g_{1}(t)$ increases with penetration depth. To decide whether or not this increase can be explained by the evanescent illumination profile alone, or if an additional viscous drag contribution has to be considered, we derived an expression for the short time behavior of $g_{1}(t)$, which takes into account both effects. A detailed discussion of this expression will be the subject of a separate publication. An outline of the deri- 


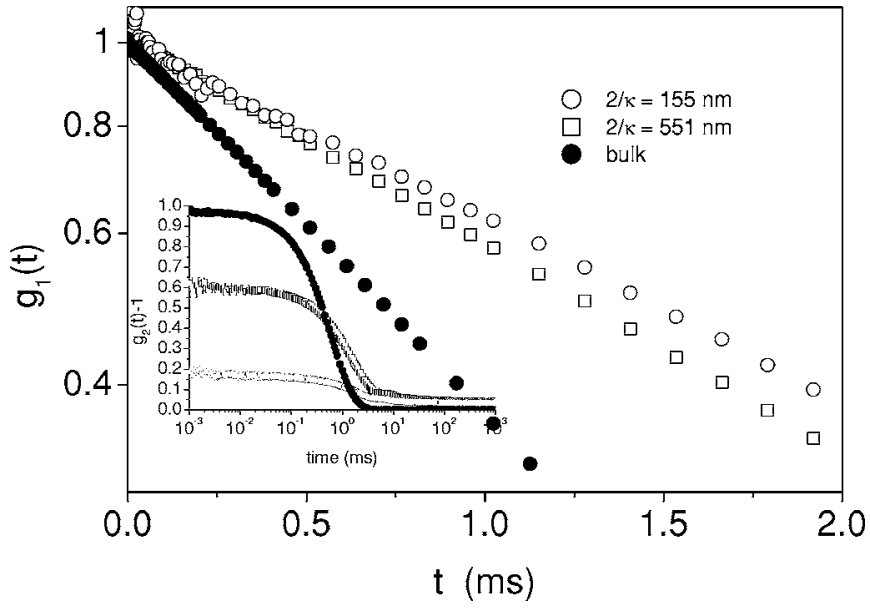

FIG. 3. Initial decay of field auto correlation functions recorded at two different penetration depths as indicated in the legend. The scattering vector components were kept constant at $Q_{\|}=0.01316 \mathrm{~nm}$ and $Q_{\perp}=0.01145 \mathrm{~nm}$. The field autocorrelation functions were calculated using Eq. (1) from the experimental intensity autocorrelation functions which are displayed in the inset over the full time range. For comparison the bulk correlation functions recorded at the same scattering vector magnitude $Q=\sqrt{Q_{\|}^{2}+Q_{\perp}^{2}}$ are also displayed.

vation is given in the following. To derive an expression for the entire TCF of the scattered field, one should consider two different diffusivities parallel and normal to the wall, $D_{\|}(z)$ and $D_{\perp}(z)$, which depend differently on the separation distance $z$ of a colloidal sphere to the wall. The equation of motion for the probability density function (PDF) $P\left(\mathbf{r}_{0}, \mathbf{r} \mid t\right)$ of finding the particle at position $\mathbf{r}$ at time $t$, which was at the position $\mathbf{r}_{0}$ at $t=0$, reads

$$
\begin{aligned}
\frac{\partial P\left(\mathbf{r}_{0}, \mathbf{r} \mid t\right)}{\partial t}= & \frac{\partial}{\partial z}\left[D_{\perp}(z) \frac{P\left(\mathbf{r}_{0}, \mathbf{r} \mid t\right)}{\partial z}\right] \\
& +D_{\|}(z)\left(\frac{\partial^{2}}{\partial x^{2}}+\frac{\partial^{2}}{\partial y^{2}}\right) P\left(\mathbf{r}_{0}, \mathbf{r} \mid t\right) .
\end{aligned}
$$

So far, this equation has not been solved analytically. We therefore choose to calculate a short time expression for $g_{1}(t)$,

TABLE I. Mean diffusivities $\left\langle D_{\|}\right\rangle$and $\left\langle D_{\perp}\right\rangle$ as a function of the penetration depth $2 / \kappa$.

\begin{tabular}{cccccc}
\hline \hline & \multicolumn{3}{c}{$\begin{array}{c}\text { From slopes } \\
\text { according to Eq. (5) }\end{array}$} & & \multicolumn{2}{c}{$\begin{array}{c}\text { From intersects } \\
\text { according to Eq. (5) }\end{array}$} \\
\cline { 1 - 3 } \cline { 5 - 6 } $\begin{array}{c}2 / \kappa \\
(\mathrm{nm})\end{array}$ & $\begin{array}{c}\left\langle D_{\|}\right\rangle \\
\left(\mathrm{nm}^{2} / \mathrm{ms}\right)\end{array}$ & $\begin{array}{c}\left\langle D_{\perp}\right\rangle \\
\left(\mathrm{nm}^{2} / \mathrm{ms}\right)\end{array}$ & & $\begin{array}{c}\left\langle D_{\|}\right\rangle \\
\left(\mathrm{nm}^{2} / \mathrm{ms}\right)\end{array}$ & $\begin{array}{c}\left\langle D_{\perp}\right\rangle \\
\left(\mathrm{nm}^{2} / \mathrm{ms}\right)\end{array}$ \\
\hline 155 & $1900 \pm 277$ & $1250 \pm 156$ & & $1880 \pm 287$ & $1200 \pm 171$ \\
234 & $2000 \pm 161$ & $1320 \pm 63$ & & $2000 \pm 168$ & $1350 \pm 78$ \\
304 & $2100 \pm 130$ & $1500 \pm 55$ & & $2280 \pm 163$ & $1500 \pm 83$ \\
551 & $2570 \pm 100$ & $1800 \pm 137$ & $2490 \pm 115$ & $1380 \pm 112$ \\
\hline \hline
\end{tabular}

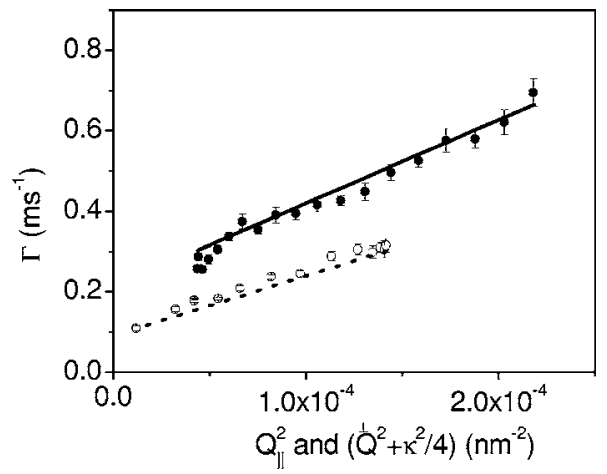

FIG. 4. Initial relaxation rates $\Gamma$ from correlation functions recorded at a penetration depth of $2 / \kappa=304 \mathrm{~nm}$. The symbol $(\bigcirc)$ relates to different values of $Q_{\perp}$ and constant $Q_{\|}$vs $\left(Q_{\perp}^{2}+\kappa^{2} / 4\right)$, while ( refers to different values of $Q_{\|}$and constant $Q_{\perp}$ vs $Q_{\|}^{2}$. The straight lines are predictions using Eq. (5).

$$
g_{1}(t)=\exp \left\{-\Gamma t+\mathcal{O}\left(t^{2}\right)\right\},
$$

where $\Gamma$ can be shown, on the basis of Eq. (2), to be equal to

$$
\begin{aligned}
\Gamma= & \frac{1}{\int_{z>R} d \mathbf{r} P(\mathbf{r}) \exp \{-\kappa z\}} \\
& \times\left\{\int_{z>R} d \mathbf{r} P(\mathbf{r}) \exp \{-\kappa z\}\left[D_{\|} Q_{\|}^{2}+D_{\perp}\left(Q_{\perp}^{2}+\frac{\kappa^{2}}{4}\right)\right]\right. \\
& \left.-\left(\frac{\kappa}{2}+\imath Q_{\perp}\right) \int_{z>R} d \mathbf{r} D_{\perp} \exp \{-\kappa z\} \frac{\partial P(\mathbf{r})}{\partial z}\right\}
\end{aligned}
$$

Here, $R$ is the radius of the colloidal spheres and $P(\mathbf{r})$ is the probability to find a particle at position $\mathbf{r}$. It is important to

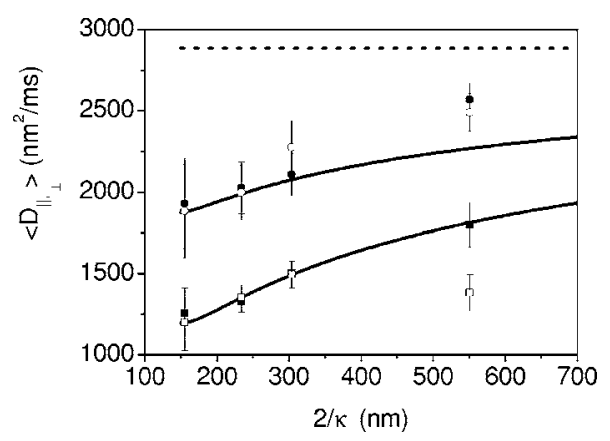

FIG. 5. Mean diffusivities calculated from slopes (full symbols) and intersects (open symbols) according to Eq. (5). $\left\langle D_{\|}\right\rangle$(bullets) and $\left\langle D_{\perp}\right\rangle$ (squares) vs penetration depth. The full lines are predictions using Eq. (7) and the dotted line represents the bulk diffusion coefficient $D_{0}$. 
note that this does not represent a cumulant expansion for $g_{1}(t)$, but the expression is exact to first order in time. Since $P(\mathbf{r})$ is constant for dilute solutions, Eq. (4) can be rewritten as

$$
\Gamma=Q_{\|}^{2}\left\langle D_{\|}\right\rangle(\kappa)+\left(Q_{\perp}^{2}+\frac{\kappa^{2}}{4}\right)\left\langle D_{\perp}\right\rangle(\kappa)
$$

where the "mean diffusivities" parallel and normal to the wall are given by

$$
\left\langle D_{\|, \perp}\right\rangle(\kappa)=D_{0} \kappa \int_{z>R} d z \exp \{-\kappa(z-R)\} / \Lambda_{\|, \perp}(z)
$$

Using Faxén's [9] and Brenner's [10,11] expressions for $\Lambda_{\|, \perp}$, the following approximations are obtained:

$$
\left\langle D_{\|}\right\rangle(\kappa) \cong D_{0}\left(\left[-\frac{9}{16} \mathcal{X}+\frac{1}{16} \mathcal{X}^{3}+\frac{15}{512} \mathcal{X}^{4}-\frac{1}{384} \mathcal{X}^{5}\right] \times \exp \{\mathcal{X}\} E_{1}(\mathcal{X})+1-\frac{3}{256} \mathcal{X}-\frac{43}{1536} \mathcal{X}^{2}-\frac{49}{1536} \mathcal{X}^{3}+\frac{1}{384} \mathcal{X}^{4}\right)
$$

and

$$
\left\langle D_{\perp}\right\rangle(\kappa) \cong D_{0}\left[1-\mathcal{X} \exp \{\mathcal{X}\} E_{1}(\mathcal{X})\right]
$$

with $E_{1}(\mathcal{X})=\int_{1}^{\infty} d z^{\prime} \exp \left\{-\mathcal{X} z^{\prime}\right\} / z^{\prime}$ and $z^{\prime}=z / R$. These approximations are accurate for $z-R>0.1 R$ and $\mathcal{X}=\kappa R \ll 1$.

To determine $\Gamma$ from experimental intensity time autocorrelation functions, their initial decay up to $t \leqslant 2 \mathrm{~ms}$ is fitted with a combination of Eqs. (1) and (3) neglecting the higherorder terms in $t$, i.e.,

$$
\begin{aligned}
g_{2}(t)= & 1+(1-A+\sqrt{1-A}) \exp \{-\Gamma t\} \\
& +[(1-\sqrt{1-A}) \exp \{-\Gamma t\}]^{2}
\end{aligned}
$$

Resulting values for the first cumulant $\Gamma$ are plotted versus $Q_{\|}^{2}$ and $\left(Q_{\perp}^{2}+\kappa^{2} / 4\right)$ in Fig. 4 for a penetration depth of $2 / \kappa=304 \mathrm{~nm}$. In both sets of data, $\Gamma$ depends linearly on $Q_{\|}^{2}$ and $\left(Q_{\perp}^{2}+\kappa^{2} / 4\right)$ as expected from Eq. (5). In the latter case the slope is evidently smaller than in the first, which shows that, close to the wall, the diffusivity of the particles is anisotropic in the range of separation distances that are probed. Corresponding results were obtained at different penetration depths. According to Eq. (5) the mean diffusivities can be determined in two ways. The slope $\partial \Gamma / \partial\left(Q_{\perp}^{2}+\kappa^{2} / 4\right)$ at constant $Q_{\|}$is $\left\langle D_{\perp}\right\rangle$ and the mean diffusivity parallel to the wall is related to the intersect by $\left\langle D_{\|}\right\rangle=\Gamma\left(Q_{\perp}=0\right) / Q_{\|}^{2}$. Accordingly, $\partial \Gamma / \partial Q_{\|}^{2}$ at constant $Q_{\perp}$ is $\left\langle D_{\|}\right\rangle$and $\left\langle D_{\perp}\right\rangle=\Gamma\left(Q_{\perp}=0\right) /\left(Q_{\|}^{2}+\kappa^{2} / 4\right)$. The observed mean diffusivities for four various penetration depths $2 / \kappa$ are listed in Table I and plotted in Fig. 5. Approximate predictions for $\left\langle D_{\|, \perp}\right\rangle$ vs $2 / \kappa$, according to Eq. (7), are displayed for comparison. The effect of the wall viscous drag is best illustrated by eliminating it from Eqs. (3)-(7) by setting $\Lambda_{\|, \perp}=1$. Then $\left\langle D_{\|}\right\rangle=\left\langle D_{\perp}\right\rangle=D_{0}$ and the short time expansion for the field correlation function becomes

$$
g_{1}^{0}(t)=\exp \left\{-\left[D_{0} Q_{\|}^{2}+D_{0}\left(Q_{\perp}^{2}+\kappa^{2} / 4\right)\right] t\right\}
$$

which is the expression obtained by Lan et al. [1]. This predicts that the $Q$-dependence of the initial decay of the correlation function should reflect the bulk diffusion coefficient $D_{0}$ alone. Differently, we observe for all penetration depths that are probed that the mean diffusivities are significantly smaller than $D_{0}$. At $2 / \kappa=155 \mathrm{~nm}$ we find that the mean diffusivity parallel to the interface is about half the bulk diffusion coefficient and $\left\langle D_{\perp}\right\rangle \approx 0.3 D_{0}$. As intuitively expected, the deviations become smaller with increasing penetration depth. At the highest experimental penetration depth we find $\left\langle D_{\|}\right\rangle \approx 0.9 D_{0}$ while $\left\langle D_{\perp}\right\rangle$ is roughly half of $D_{0}$. Note that the increase of the mean diffusivities, $\left\langle D_{\|, \perp}\right\rangle$, with penetration depth, is less steep as compared to the diffusivities $D_{\|, \perp}(z)$ in Eq. (2). This is due to the different weighting of the particles by the evanescent illumination. Particles closer to the surface are less mobile but experience a higher strength of the evanescent field. Therefore, they contribute with a higher weight to the scattered intensity than particles further away from the wall, which have a higher mobility. For this reason the averaged diffusivities will reach the value of the bulk diffusion only for values of $2 / \kappa R$ significantly larger than ten. In fact Eq. (7) predicts that $\left\langle D_{\|}\right\rangle>0.95 D_{0}$ only for $2 / \kappa R>70$, which is not accessible with particles larger than roughly $10 \mathrm{~nm}$ in radius.

In conclusion we show for the first time, experimentally, that the effect of a wall viscous drag gives rise to an anisotropy of the mobility of Brownian particles as small as $R$ $=85 \mathrm{~nm}$. With our setup, it is possible to measure mean diffusivity parallel and normal to the wall independently. We find that the wall drag effect has a pronounced effect on the diffusive properties of colloidal spheres, even for extended distances away from the wall many times the size of the colloidal spheres. In addition we have derived an expression for the short-time expansion of the intensity time autocorrelation function with evanescent wave illumination with the inclusion of wall drag effects. This expression is essential to unambiguously interpret experimental data. 
[1] K. H. Lan, N. Ostrowsky, and D. Sornette, Phys. Rev. Lett. 57, 17 (1986).

[2] N. Garnier and N. Ostrowsky, J. Phys. II 1, 1221 (1991).

[3] M. I. M. Feitosa and O. N. Mesquita, Phys. Rev. A 44, 6677 (1991).

[4] M. Hosoda, K. Sakai, and K. Takagi, Phys. Rev. E 58, 6275 (1998).

[5] H. Matsuoka, H. Morikawa, S. Tanimoto, A. Kubota, Y. Naito, and H. Yamaoka, Colloid Polym. Sci. 276, 349 (1998).

[6] B. Loppinet, G. Petekidis, and G. Fytas, Langmuir 14, 4958 (1998).

[7] G. Fytas, S. H. Anastasiadis, R. Seghrouchni, D. Vlassopoulos, J. Li, B. J. Factor, W. Theobald, and C. Toprakcioglu, Science 274, 2041 (1996).
[8] G. E. Yakubov, B. Loppinet, H. Zhang, J. Rühe, R. Sigel, and G. Fytas, Phys. Rev. Lett. 92, 115501 (2004).

[9] H. Faxén, Ark. Mat., Astron. Fys. 17, 1 (1923).

[10] H. Brenner, Chem. Eng. Sci. 16, 242 (1961).

[11] A. J. Goldman, R. G. Cox, and H. Brenner, Chem. Eng. Sci. 22, 637 (1967).

[12] Z. Adamcyzk, M. Adamcyzk, and T. G. M. Van De Ven, J. Colloid Interface Sci. 96, 204 (1983).

[13] N. Lecoq, F. Feuillebois, N. Anthore, R. Anthore, F. Bostel, and C. Petipas, Phys. Fluids A 5, 3 (1993).

[14] K. D. Kihm, A. Banerjee, C. K. Choi, and T. Tagaki, Exp. Fluids 37, 811 (2004).

[15] A. Banerjee and K. D. Kihm, Phys. Rev. E 72, 042101 (2005).

[16] B. Lin, J. Yu, and S. A. Rice, Phys. Rev. E 62, 3909 (2000). 